\title{
BMJ Open The provision of medical assistance in dying: protocol for a scoping review
}

\author{
Simon J W Oczkowski, ${ }^{1,2}$ Ian Ball, ${ }^{3}$ Carol Saleh, ${ }^{4}$ Gaelen Kalles, ${ }^{2}$ \\ Anatoli Chkaroubo, ${ }^{2}$ Mike Kekewich, ${ }^{5}$ Paul Miller, ${ }^{2,6}$ Marianne Dees, ${ }^{7}$ Andrea Frolic ${ }^{2}$
}

To cite: Oczkowski SJW, Ball I, Saleh C, et al. The provision of medical assistance in dying: protocol for a scoping review. BMJ Open 2017;7:e017888. doi:10.1136/ bmjopen-2017-017888

- Prepublication history and additional material are available online. To view these files please visit the journal online (http://dx. doi.org/10.1136/bmjopen-2017017888).

Received 23 May 2017

Revised 19 June 2017

Accepted 22 June 2017

CrossMark

${ }^{1}$ Division of Critical Care, Department of Medicine, McMaster University, Hamilton, Canada

${ }^{2}$ Hamilton Health Sciences, Hamilton, Canada

${ }^{3}$ Division of Critical Care Medicine, Department of Epidemiology and Biostatistics Western University, London,

Canada

${ }^{4}$ Department of Medicine, McMaster University, Hamilton, Canada

${ }^{5}$ Department of Clinical and Organizational Ethics, The

Ottawa Hospital, Ottawa, Canada

${ }^{6}$ Division of Emergency

Medicine, Department of

Medicine, McMaster University, Hamilton, Canada

${ }^{7} \mathrm{IQ}$ Healthcare, Radboud Institute for Health Sciences,

Radboud University Medical

Center, Nijmegen, The

Netherlands

Correspondence to

Dr Simon J W Oczkowski;

oczkowsj@mcmaster.ca

\section{ABSTRACT}

Introduction Medical assistance in dying (MAID), a term encompassing both euthanasia and assisted suicide, was decriminalised in Canada in 2015. Although Bill C-14 legislated eligibility criteria under which patients could receive MAID, it did not provide guidance regarding the technical aspects of providing an assisted death. Therefore, we propose a scoping review to map the characteristics of the existing medical literature describing the medications, settings, participants and outcomes of MAID, in order to identify knowledge gaps and areas for future research.

Methods and analysis We will search electronic databases (MEDLINE, EMBASE, CINAHL, CENTRAL, PsycINF0), clinical trial registries, conference abstracts, and professional guidelines and recommendations from jurisdictions where MAID is legal, up to June 2017. Eligible report types will include technical summaries, institutional policies, practice surveys, practice guidelines and clinical studies. We will include all descriptions of MAID provision (either euthanasia or assisted suicide) in adults who have provided informed consent for MAID, for any reason, including reports where patients have provided consent to MAID in advance of the development of incapacity (eg, dementia). We will exclude reports in which patients receive involuntary euthanasia (eg, capital punishment). Two independent investigators will screen and select retrieved reports using pilot-tested screening and eligibility forms, and collect data using standardised data collection forms. We will summarise extracted data in tabular format with accompanying descriptive statistics and use narrative format to describe their clinical relevance, identify knowledge gaps and suggest topics for future research. Ethics and dissemination This scoping review will map the range and scope of the existing literature on the provision of MAID in jurisdictions where the practice has been decriminalised. The review will be disseminated through conference presentations and publication in a peer-reviewed journal. These results will be useful to clinicians, policy makers and researchers involved with MAID.

\section{INTRODUCTION}

\section{Background}

In its 2015 ruling Carter v Canada, the Supreme Court of Canada (SCC) struck down the criminal prohibition on assisting individuals in suicide, if physicians deemed such individuals to be competent adults with
Strengths and limitations of this study

This will be the first scoping literature review on the provision of medical assistance in dying (MAID).

- The search strategy includes a comprehensive and systematic search of five electronic databases, and grey literature, including conference proceedings, clinical trial registries, and clinical protocols from jurisdictions where MAID has been decriminalised.

- Although the study will provide a descriptive overview of how medical assistance in dying may be provided, no formal assessment of the quality of evidence for any given approach will be conducted.

- Though this review will not provide recommendations for how to provide medical assistance in dying, an overview of current practices and knowledge gaps may still inform clinicians, policy makers and researchers working in this area.

a 'grievous and irremediable medical condition' causing 'enduring suffering intolerable to the individual'. ${ }^{1}$ The SCC suspended the ruling for 1year to provide the Canadian federal government with time to develop a legislative framework for medical assistance in dying (MAID). ${ }^{2}$ In June 2016, the federal government passed Bill C-14, which decriminalised assisted dying for capable patients with intolerable suffering for whom death was 'reasonably foreseeable'.

\section{Study rationale}

Although Bill C-14 legislated eligibility criteria under which patients could receive MAID, the law did not provide clinicians or organisations with guidance regarding the technical aspects of providing MAID, including fundamental issues as whether it should be in the form of assisted suicide (in which a person self-administers a lethal medication prescribed and provided by a healthcare professional) or euthanasia (in which a person receives a lethal dose of medication at the hands of a healthcare professional). As a result, Canadian clinicians and organisations have struggled with many practical questions about providing MAID, including: 
1. Should MAID be provided in the form of assisted suicide, euthanasia or a combination of the two?

2. Which pharmaceuticals, doses and routes of administration should be used for MAID?

3. Should MAID provision take place in the community, institutional settings, or in dedicated, expert centres?

4. What is the appropriate role, scope of practice and training of MAID providers?

5. How should patients' families be involved and supported in the provision of MAID?

Given concerns about variation in consistency and quality of MAID, including the possibility of technical problems with medication administration, and the potentially high impact of the practice on patients, families and healthcare providers, there is an urgent need to develop an evidence base to guide the provision of MAID. ${ }^{45}$ While several other jurisdictions permit MAID in the form of assisted suicide (Switzerland, and the American states of Oregon, Montana, Washington, California, Colorado, Vermont, Washington DC), euthanasia (Columbia), or both (Belgium, the Netherlands and Luxembourg), there is little readily available evidence to assist Canadian clinicians and organisations in addressing these fundamental questions about providing MAID. ${ }^{46}$

Therefore, we propose a scoping review on the provision of MAID from all jurisdictions where medically assisted dying is not illegal, in order to determine the range, scope and content of the existing medical literature on the provision of MAID in consenting adults.

\section{Study objectives}

1. To describe the existing medical literature on the provision of MAID

2. To summarise the existing medical literature and provide an overview of the technical aspects of MAID provision (including pharmaceuticals and procedures; location of provision; the role, scope of practice and training of healthcare professionals; role of patients' families; rates of adverse events)

3. To identify evidence gaps to guide future research in MAID

\section{METHODS AND ANALYSIS}

The methods of this scoping review protocol are based on those described in the Joanna Briggs Institute Reviewer's Manual. $^{7}$

\section{Inclusion and exclusion criteria}

As opposed to a systematic review, the selection of studies and reports in a scoping review is an iterative process, and inclusion and exclusion criteria may undergo revision as the review progresses, taking into account findings which emerge during the course of the review. ${ }^{7-9}$ In this protocol, we outline our initial inclusion and exclusion

Table 1 Inclusion and exclusion criteria

\begin{tabular}{|c|c|c|}
\hline & Inclusion criteria & Exclusion criteria \\
\hline \multirow{5}{*}{ Types of sources } & Institutional policy & \\
\hline & $\begin{array}{l}\text { Clinical practice guideline/ } \\
\text { recommendation }\end{array}$ & \\
\hline & Observational study & \\
\hline & Clinical trial & \\
\hline & Other (describe) & \\
\hline Types of patients & Adults (age $>18$ years) & $\begin{array}{l}\text { Patients receiving involuntary euthanasia (capital } \\
\text { punishment) }\end{array}$ \\
\hline \multirow[t]{4}{*}{ Types of interventions } & $\begin{array}{l}\text { Provision of assisted suicide or } \\
\text { euthanasia with involvement of a } \\
\text { healthcare professional (physician, } \\
\text { nurse, pharmacist, etc) }\end{array}$ & $\begin{array}{l}\text { Assisted suicide or euthanasia without involvement of a } \\
\text { health professional }\end{array}$ \\
\hline & & Description of assessment/ eligibility for MAID alone \\
\hline & & Description of ethics or acceptability of MAID \\
\hline & & $\begin{array}{l}\text { Non-MAID end-of-life practices, includingwithdrawing/ } \\
\text { withholding treatments; palliative sedation; or palliative care }\end{array}$ \\
\hline
\end{tabular}

MAID, medical assistance in dying. 
criteria (table 1), while any changes made during the course of the review will be described in the final review manuscript.

\section{Types of participants}

We will include reports which include adult (age $>18$ years) patients who have provided informed consent for MAID in the form of either assisted suicide or euthanasia, for any reason, or are intended for use with such patients. We will include studies where patients have provided informed consent to MAID in advance of the development of incapacity (eg, advanced directives for MAID), but exclude reports in which patients receive euthanasia without having provided informed consent (eg, execution).

\section{Types of interventions}

We will include reports which describe the provision of MAID by either assisted suicide or euthanasia, using any method, in any location. We will exclude reports where patients receive assisted suicide or euthanasia without the involvement of a healthcare professional such as a physician, nurse or pharmacist; reports which solely describe the assessment of patient eligibility for MAID; and descriptions of public or healthcare provider opinions about acceptability or ethics of MAID. We will also exclude reports describing other end-of-life practices, including withholding or withdrawing of life-sustaining treatment; palliative sedation or unintentional hastening of death via medications for symptom management (eg, the doctrine of double effect), unless such reports also include separate descriptions of MAID.

\section{Types of sources}

We will include technical reports, institutional policies, practice surveys, clinical practice guidelines and clinical studies (case reports, observational studies or clinical trials). Opinion pieces/letters will be excluded. We will impose no restrictions based on methodological quality, study location, language or publication date.

\section{Search strategy}

We will conduct systematic searches of multiple databases, including MEDLINE, EMBASE, CINAHL, CENTRAL and PsycINFO from database inception to June 2017 for the concept of MAID ("[medical] aid [assistance] in dying,' 'euthanasia,' 'assisted suicide,' '[physician] assisted dying,' '[physician] assisted death,' 'end of life choice') and the concept of medication administration ('practice patterns,' 'drug administration,' 'medication management,' drug utilisation,' 'drug therapy') (available as an online supplementary file 1). The electronic search will be supplemented by extensive grey literature searches, including clinical trial databases, conference abstracts from palliative care conferences (Canadian Hospice Palliative Care Conference, International Congress on Palliative Care), technical reports of MAID protocols and institutional policies for MAID. In jurisdictions where MAID is legal, we will contact professional groups (eg, medical associations), as well as government agencies which monitor and regulate healthcare (eg, ministries of health) in order to obtain protocols and reports describing the provision of MAID. The search will be complete by 30 June 2017 .

\section{Selection process}

Report eligibility will be determined in two stages: first by screening of titles and abstracts, and second by full-text screening. Two investigators (CS, SJWO) will pilot-test the screening and eligibility forms on the first 100 abstracts and the first 10 full-text reports in order to ensure consistent application of inclusion and exclusion criteria at each stage. Following pilot-testing and completion of any necessary modifications to the screening and eligibility forms, the same two investigators will independently review each report's eligibility for inclusion in the review. In the event of disagreement over report eligibility which cannot be resolved by discussion between the two investigators, a third investigator will make the final determination of eligibility. To provide a measure of the consistency of application of the inclusion and exclusion criteria at each stage, a weighted $\mathrm{K}$ statistic will be calculated as a measure of inter-rater reliability. ${ }^{10}$

If during the course of the review, the investigators believe that a change to the inclusion or exclusion criteria is warranted, this will be discussed with the entire investigative team for review and approval, to ensure that the proposed changes are consistent with the study objectives. Any such changes will be clearly delineated in the final review manuscript in order to ensure methodological transparency.

\section{Extraction of results}

Data will be collected by two investigators (CS, SJWO) using structured data extraction forms. An initial set of data items is listed below (table 2), however the final set of data items to be collected may change as review progresses, based on the data contained in the included reports. The two investigators will independently chart data from the first five included reports to pilot-test the data extraction form, thereby ensuring consistent and comprehensive data collection. Following pilot-testing and, if necessary, modification of the data extraction forms, the two investigators will continue with duplicate data extraction. As the review progresses, the investigators will compare and discuss the extracted data, and consider updating the data extraction forms to ensure that the collected data is consistent with the review's objectives. The initial data collection forms will collect data related to three major concepts: report characteristics; methods of MAID provision (medications, locations, participants); and MAID outcomes.

\section{Presentation of results}

We will organise the collected data according to the three major concepts listed above (report characteristics; MAID provision; and MAID outcomes). We will summarise the 
Table 2 Data collection items

\begin{tabular}{|c|c|}
\hline Report characteristics & Description \\
\hline Author(s) & Profession and/or specialisation \\
\hline \multicolumn{2}{|l|}{ Year of publication } \\
\hline Report type & $\begin{array}{l}\text { Technical report, practice survey, clinical practice guideline, observational study, } \\
\text { clinical trial, other (describe) }\end{array}$ \\
\hline Report citation & Primary documents on which the report is based (if relevant) \\
\hline MAID provision: medications & Description \\
\hline Pharmaceutical used & $\begin{array}{l}\text { Each pharmaceutical name, dose, route, frequency, speed of administration, } \\
\text { stated or inferred purpose of each medication (eg, anxiolytic, sedation, pain } \\
\text { control, antiemetic, paralytic) and frequency of use (optional vs obligatory); } \\
\text { alternative medications in case of allergy }\end{array}$ \\
\hline MAID provision: location & Description \\
\hline Location of provision & $\begin{array}{l}\text { Home, hospital, hospice, other, nursing home's psychiatric institutions provider's } \\
\text { profession or specialisation, self administration or euthanasia? }\end{array}$ \\
\hline MAID provision: participants & Description \\
\hline Role of healthcare provider(s) & Profession, training/expertise, role in assisted dying \\
\hline Role of families & Training/preparation; follow-up care; bereavement care \\
\hline Safety checks and documentation & $\begin{array}{l}\text { eg, use of a checklist; confirmation of consent; backup medications available, and } \\
\text { so on }\end{array}$ \\
\hline
\end{tabular}

MAID, medical assistance in dying.

report characteristics, including date of publication, publication type and geographical origin of publication in a table with accompanying univariate descriptive statistics (eg, frequency, proportion) in order to provide an overview of the characteristics of the existing medical literature on the provision of MAID.

We will summarise data about the provision of MAID and about MAID outcomes in tables categorised as follows: pharmaceuticals and equipment; location of provision; personnel; documentation; and aftercare, with accompanying descriptive statistics of frequency or proportion for categorical data, and mean and SD or median and IQR for continuous data. The tables will summarise the collected data, for assisted suicide and euthanasia separately, where appropriate (eg, pharmaceuticals, personnel). We will synthesise this information in a narrative format, describing the type and range of the available evidence and its relevance to the five questions described in the study rationale, above. Though a formal appraisal of the quality (certainty) of the evidence is not routinely conducted in a scoping review, ${ }^{7}$ we will comment on the reliability and trustworthiness of the available evidence, based on the methods of each report and the consistency, or lack of consistency, of results between reports. We will summarise the data's potential relevance to the provision of MAID in Canada, as compared with other clinical and legal contexts. In doing so, we will identify knowledge gaps and formulate topics for future research.

\section{ETHICS AND DISSEMINATION}

This scoping review will provide a comprehensive description of the range and scope of the existing literature on the provision of MAID, and a summary of the technical aspects of providing MAID. We will describe the relevance of the existing literature to the provision of MAID 
in Canada, and identify knowledge gaps and topics for future research. The results of the review will be submitted for presentation as a conference abstract, and publication in a peer reviewed journal.

Twitter@Simon_0cz

Acknowledgements SJWO is supported by an Internal Career Award from the Department of Medicine at McMaster University.

Contributors SJWO conceived the idea for the project and developed the initial draft of the manuscript. All authors developed the review methodology and edited and revised the manuscript for essential content and formatting details, and approved the final version of the manuscript for submission. SJWO and CS will conduct the data collection, data extraction for the review. All authors will contribute to the analysis of the review data.

Funding This review was supported by a grant from the Hamilton Academic Health SciencesOrganization Innovation Fund.

Competing interests None declared.

Patient consent Not applicable.

Provenance and peer review Not commissioned; externally peer reviewed.

Data sharing statement Any unpublished information from the final review will be made available upon request.

Open Access This is an Open Access article distributed in accordance with the Creative Commons Attribution Non Commercial (CC BY-NC 4.0) license, which permits others to distribute, remix, adapt, build upon this work non-commercially, and license their derivative works on different terms, provided the original work is properly cited and the use is non-commercial. See: http://creativecommons.org/ licenses/by-nc/4.0/

(C) Article author(s) (or their employer(s) unless otherwise stated in the text of the article) 2017. All rights reserved. No commercial use is permitted unless otherwise expressly granted.

\section{REFERENCES}

1. Carter v. Canada Attorney General. SCC, 2015.

2. Attaran A. Unanimity on death with dignity--legalizing physicianassisted dying in Canada. N Engl J Med 2015;372:2080-2.

3. First Session of the Parliament of Canada. Bill C-14: an Act to amend the Criminal Code and to make related amendments to other acts (medical assistance in dying). 2016.

4. Hendin H. Physician-Assisted Suicide and Euthanasia in the Netherlands. J Am Med Assoc 1720;1997:277.

5. Groenewoud JH, van der Heide A, Onwuteaka-Philipsen BD, et al. Clinical problems with the performance of euthanasia and physicianassisted suicide in The Netherlands. N Engl J Med 2000;342:551-6.

6. Veldink JH, Wokke JH, van der Wal G, et al. Euthanasia and physician-assisted suicide among patients with amyotrophic lateral sclerosis in the Netherlands. N Engl J Med 2002;346:1638-44.

7. Peters M, Godfrey C, Mclnerney P, et al. The Joanna Briggs Institute Reviewers' Manual 2015: Methodology for JBI Scoping Reviews. 2015.

8. Arksey H, O'Malley L. Scoping studies: towards a methodological framework. Int J Soc Res Methodol 2005;8:19-32.

9. Levac D, Colquhoun H, O'Brien KK. Scoping studies: advancing the methodology. Implement Sci 2010;5:69.

10. Viera AJ, Garrett JM. Understanding interobserver agreement: the kappa statistic. Fam Med 2005;37:360-3. 